\title{
Development of smart online partial discharge monitoring system for medium voltage power cable
}

\author{
A. Z. Abdullah ${ }^{1}$, M. Isa ${ }^{2}$, M. N. K. H. Rohani ${ }^{3}$, S. A. S. Jamalil ${ }^{4}$, A. N. N. Abdullah ${ }^{5}$, N. Azizan ${ }^{6}$ \\ 1,2,3,5 High Voltage Transient \& Insulation Health (HVTrans) Group, Centre of Excellence for Renewable Energy \\ (CERE), School of Electrical System Engineering, Universiti Malaysia Perlis (UniMAP), Malaysia \\ ${ }^{4,6}$ Faculty Engineering Technology, Universiti Malaysia Perlis (UniMAP), Malaysia
}

\begin{tabular}{l}
\hline \hline Article Info \\
\hline Article history: \\
Received Apr 1, 2019 \\
Revised Jun 30, 2019 \\
Accepted Jul 11, 2019 \\
\hline
\end{tabular}

Keywords:

LabVIEW

Monitoring system

Partial discharge

Rogowski coil

Underground cable

\begin{abstract}
This paper presents, the development of smart online partial discharge (PD) monitoring system for medium voltage (MV) underground power cable. PD monitoring is the highly efficient tool to monitor insulation degradation for high voltage (HV) equipment in order to avoid failures or breakdown. Selection of improved technology and performance of PD detection sensor, effective measurement technique and smart user friendly of graphical user interface (GUI) system are contributed towards the development of efficient monitoring system. This paper addresses three main aspects which are needed in completing the monitoring system. They are, the use of Rogowski coil (RC) as detection sensor, processing unit using Alterra board and integrated with GUI PD monitoring system for underground cable using LabVIEW. The monitoring system is compared to the conventional method with the PD signal used is measured from the real on-site measurement in order to analyse its performance. The analysis is performed in MATLAB and LabVIEW software's environment and the maximum peak of PD signal is enabled to view using GUI which complete with the location information of PD source. Furthermore, this paper has contributed to solve the problem in selection the simplified and practical approach for PD sensor and monitoring system. In the perspective of automated condition monitoring, this smart online PD monitoring system provides a complete solution towards latest industrial revolutions
\end{abstract}

Copyright (c) 2019 Institute of Advanced Engineering and Science. All rights reserved.

\section{Corresponding Author:}

A. Z. Abdullah,

School of Electrical System Engineering,

Universiti Malaysia Perlis (UniMAP),

Kampus Pauh Putra, 02600 Arau, Perlis, Malaysia.

Email: zaidiabdullah@unimap.edu.my

\section{INTRODUCTION}

Condition monitoring technology plays a major role to keep the electrical supply network in reliable operation. The electrical distribution over the world are operated at 24 hours and 7 days a week simultaneously. Therefore, various electrical power components faced with environmental stresses [1]. Condition monitoring in power system requires best infrastructure of components and equipment which is reliable when effective maintenance applied [2]. Early information for potential breakdown in on-line mode especially for PD in HV equipment is consider crucial [3]. Therefore, the utility providers keep on striving determine solutions to improve the reliability in continuity of the power supply [4].

MV underground power cable is among the key component in distribution networks. Normally, this component is undergoing stress condition includes, electrical, thermal, mechanical and ambient, stresses, that significantly toward to the degradation of its insulation $[5,6]$. The degradation insulation in MV underground power cable is highly proven originated from PD activities. PD is a localize discharge that only partially 
bridges the insulation between the conductor and which may or may not occur adjacent to a conductor [7]. The high accuracy of PD monitoring system is needed to capture PD activity since it occurs in short duration of current pulses, normally for only a few nanoseconds [8]. Referring to the data from [9], PD usually occurs before breakdown happened indicate that PD monitoring can be a good system to installed. The data collected through PD monitoring system also can give a real time of insulation system healthy. Thus, the reliability, efficiency, equipment safety and cost can be improved.

Traditional method is using off-line measurement where it will interrupt the normal operation. There will be more economical, if the equipment can be tested without interrupt the whole system. Thus, online PD monitoring is the best way to ensure the performance of PD analysis efficiently [10]. The real-time PD monitoring and activity using simulation LabVIEW software is used for further processed [11]. The biggest advantage of a GUI is that it works without prior knowledge and it is the most important aspect for any application [12-14].

Currently, conventional PD sensor are saturated, big size, bulky, low sensitivity and high cost such as High Frequency Current Transformer (HFCT) and Coupling Capacitor (CC) compared to Rogowski coil (RC) [15]. PD activity will be measured thru current pulse when RC is placed around the cable with supply make it convenient to use [16]. While during on-line measurement also have limitation such as need manually collecting data and require a lot of time and human force [17-20]. Therefore, in this paper, an attempt is made to develop a smart online partial discharge monitoring system for high voltage underground cable complete with hardware and graphical user interface (GUI). The peak detector and thresholding technique were a used and indicate good result compare to others,

\section{RESEARCH METHODS}

This monitoring system is developed based on the combination of hardware, software and PD measurement setup at on-site. The RC is used as a measuring sensor for the measurement. Figure 1 shows the complete diagram for PD monitoring system at MV distribution network system. The system is comprised with three RC sensors, labelled as RC set A, RC set B and RC set C, looped along $5 \mathrm{~km}$ power cable. Each $\mathrm{RC}$ is integrated with nano5 which is able to transmit data to the server or cloud. Then, monitoring system using GUI which is under LabVIEW interface will always work in real time at maintenance office thru Wi-Fi connection to receive and process the data from measuring sensors. The PD activity usually start with small condition and expand rapidly if there are no prevention action taken and will end up with fault or breakdown in the whole system. Therefore, monitoring procedure must be performed in real time in order to determine the actual condition of power cable.

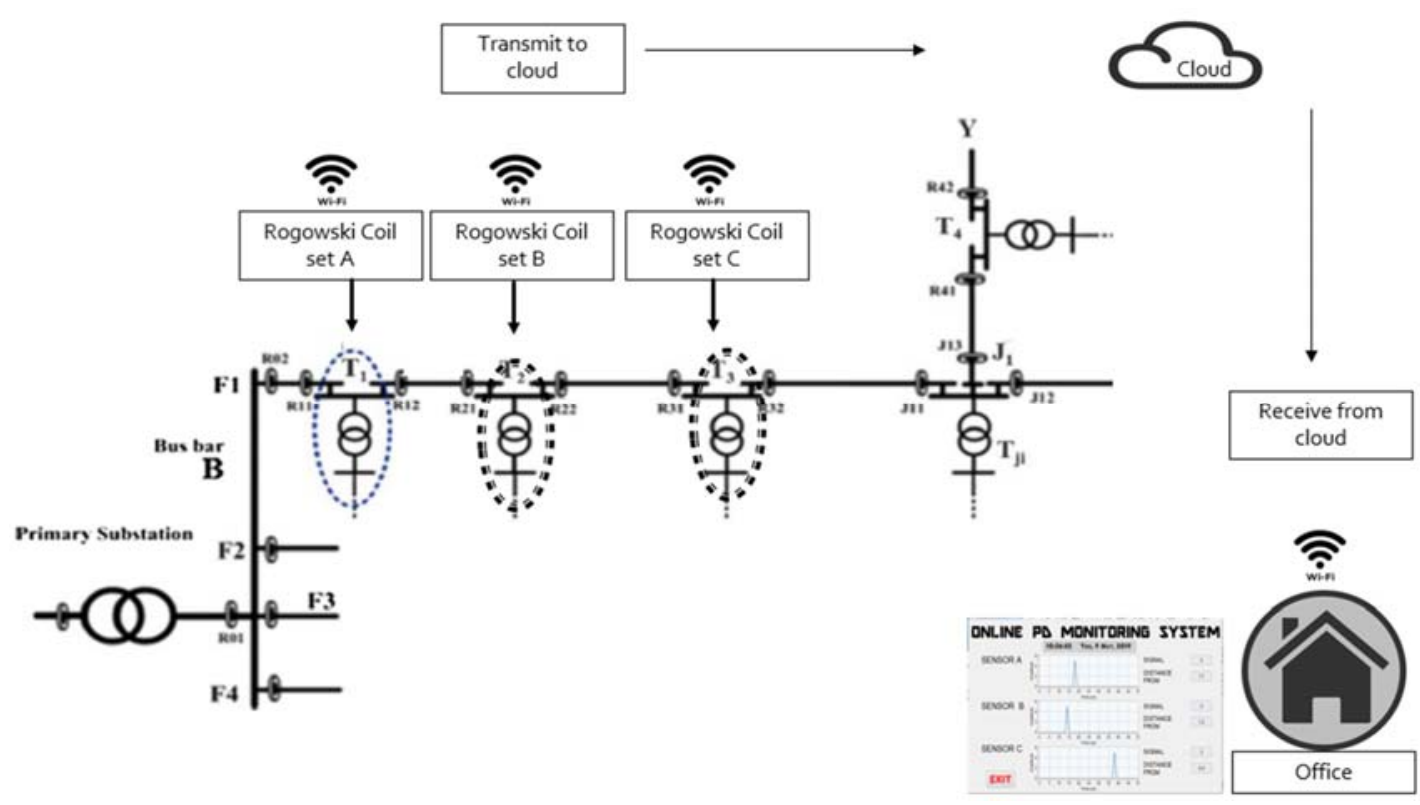

Figure 1. Diagram of wireless PD monitoring system using RC sensor 


\subsection{Sensors for Partial Detection (PD) Detection}

Table1 represents the comparison between different type of measuring sensors for PD detection. It shows that the electromagnetic detection using modified RC address many advantages in terms of price, weight, performance and installation method compared to the HFCT and CC sensors[21, 22].

Table 1. Comparison between sensor for partial discharge detection.

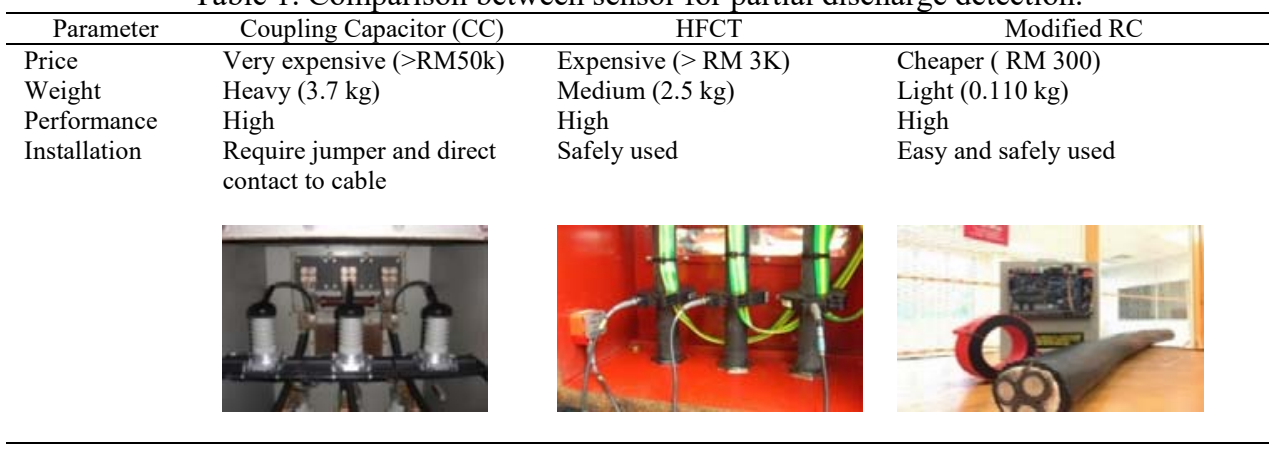

\subsection{Partial Discharge (PD) Detection Technique}

A fault detection technique was developed in order to improve accuracy and at lower cost. The peak detection flow under processing unit is as shown in Figure 2. The processing flow is started with detection counter in term of peak value setting versus time in Alterra board. The PD signal will be declared occur when the peak signal is more than threshold value set in the processing unit. The process is also confirmed by the control unit $[23,24]$. This detection applied the concept of eliminating low voltage signal as shown in Figure 3. Finally, the data is sent to the cloud through Wi-Fi. Hence, the $\boldsymbol{\Sigma} \boldsymbol{\Delta}$ ADC topology is a novel by implementing in wireless RC sensor development based on PD detection technique and become the preferred solution [19].

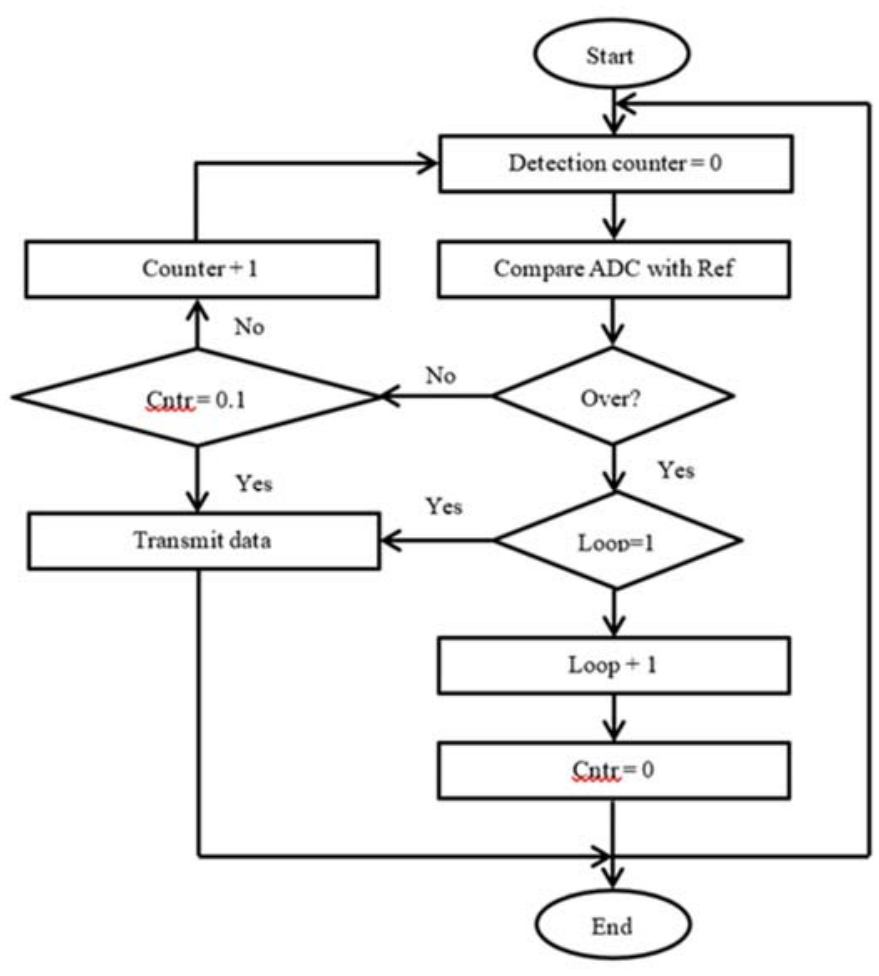

Figure 2. Flow chart of PD detection process. 


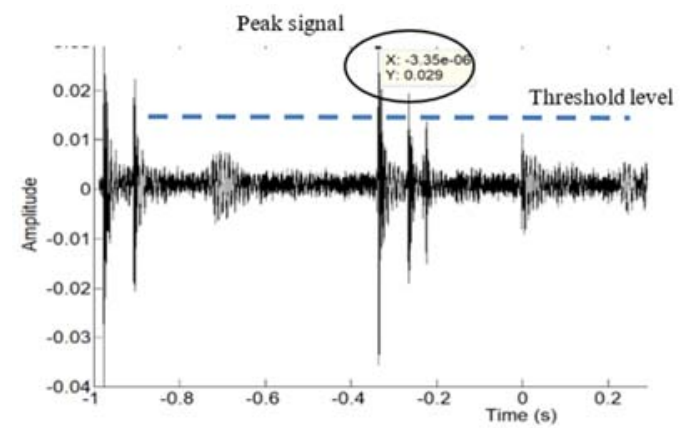

Figure 3. Peak detector and thresholding technique

Once peak signal was detected, there mathematical equations as in (1) (2) (3)and (4) was used to simulate the exact signal to fulfil PD characteristic and then use for PD localization analysis[25]. The different time arrivals between sensor $\mathrm{A}$ and $\mathrm{B}$ is represented by $t_{a b}$, and between sensor $\mathrm{B}$ and sensor $\mathrm{C}$ is represented by $t_{c b}$ respectively.

$$
\begin{aligned}
& t_{a b}=t_{a}-t_{b} \\
& t_{c b}=t_{c}-t_{b} \\
& X=\frac{1}{4} L\left(\frac{t_{a b}}{t_{c b}}+1\right) \\
& s(t)=A\left[e^{-a_{1} t} \cos \left(w_{d} t-\varphi\right)-e^{-a_{2} t} \cos (\varphi)\right]
\end{aligned}
$$

where $A$ is 0.01 magnitude coefficient

$\mathrm{a}_{1}=1 \mathrm{Ms}^{-1}, \mathrm{a}_{2}=10 \mathrm{Ms}^{-1}, \varphi=\tan ^{-1}\left(\mathrm{w}_{\mathrm{d}} / \mathrm{a}_{2}\right), \mathrm{w}_{\mathrm{d}}=2 \pi f d, \mathrm{fd}=1 \mathrm{MHz}$

\subsection{Graphical User Interface (GUI) for Partial Discharge Monitoring System}

Figure 4 shows the internal block diagram of PD monitoring system under LabVIEW software environment. Several types of graphical functions were used, such as formula waveform vi, subtraction and multiplication, exponential, spectral measurement vi to complete the circuit connection. Besides, the specific input from measurement of sensor $\mathrm{A}, \mathrm{B}, \mathrm{C}$ will analyse and perform using algorithm to produce signal that give PD signal with the highest peak.

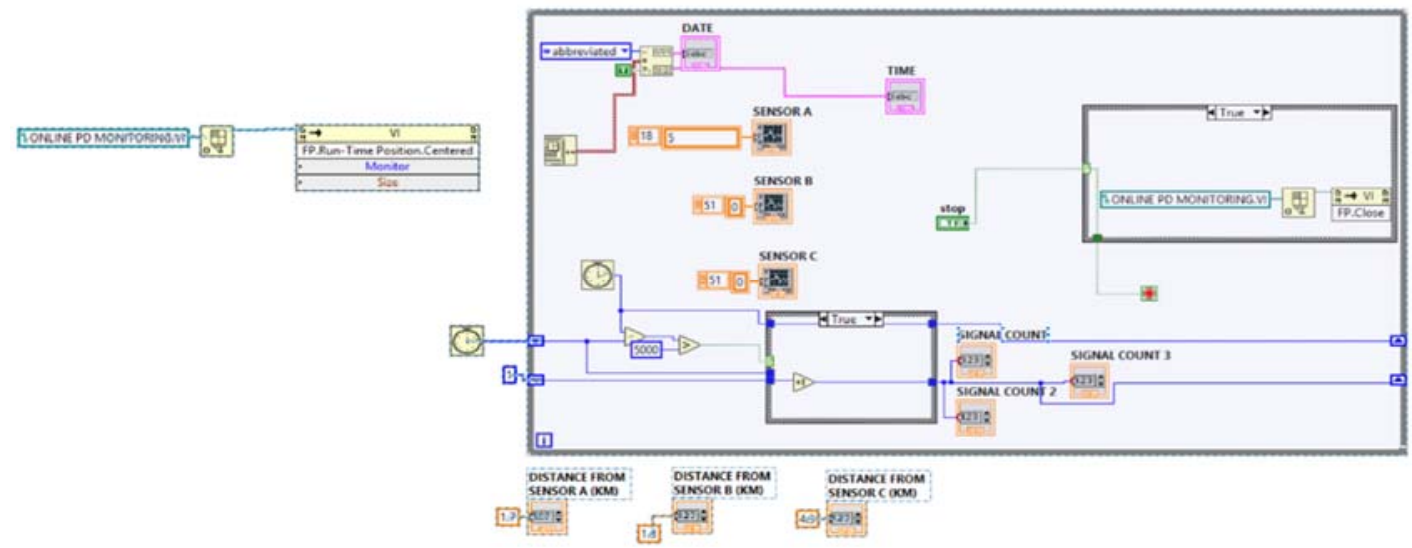

Figure 4. Block diagram of PD monitoring system

\subsection{Hardware Development for Partial Discharge (PD) Monitoring System}

Development of smart online partial discharge monitoring system for medium ... (A. Z. Abdullah) 
Figure 5(a) shows the complete of PD monitoring system hardware for $11 \mathrm{kV}$ power cable. The measuring sensor developed can be used for the power cable size up to $180 \mathrm{~mm}^{2}$. While Figure 5(b) represents the schematic diagram for the monitoring system developed. RC sensor is the main component for the PD detection circuit. and the Altera DE2-115 board system for the processing unit. The nano5 unit is integrated with each RC sensor in order to transmit signal through Wi-Fi to the cloud. The algorithm is integrated on Alterra board which complete with PD peak detector, analogue to digital conversion and Wi-Fi receiver.

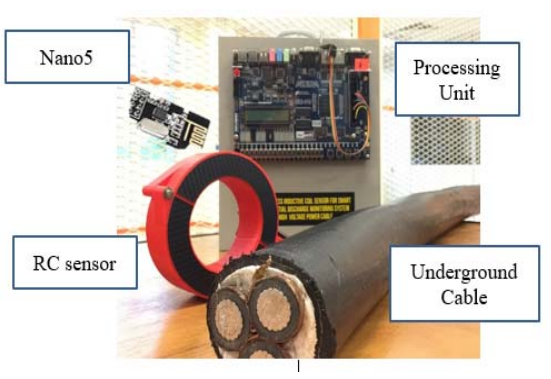

(a)

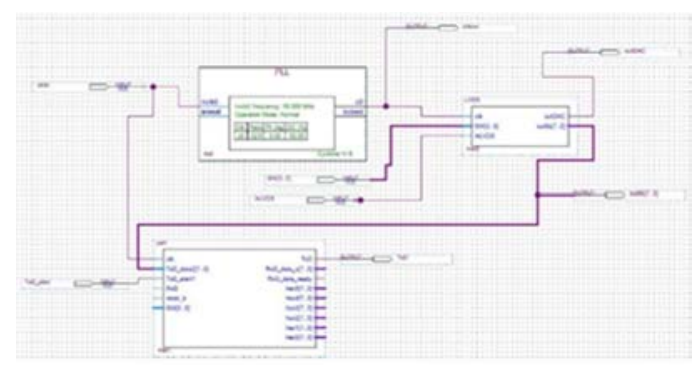

(b)

Figure 5. (a) Smart online PD monitoring system hardware, (b) Schematic diagram.

\section{RESULTS AND ANALYSIS}

Figure 6 shows the actual PD signal waveform measured using RC at site. Meanwhile Figure 7 shows the peak voltage of the highest PD signal measured from the measurement. This waveform is captured by RC and trimmed by the processing unit in order to identify the PD signal. The processing unit helps to identify the highest PD signal because the peak signal is set to triggered at certain level, system make easier to identify the highest PD signal. The selection of N-bit resolution value result is $0.029 \mathrm{~V}$. Then, Figure 8 shows the conversion from analogue signal to digital waveform. Although, the higher resolutions have a better in minimum digital voltage, but it is difficult to identify the greatest peak voltage due to the value is really close, compared with 16-bit voltage resolutions. The analog signal and digital signal is compared in term of the maximum peak signal by following (4) where, the output of Altera DE0-nano board is $3.3 \mathrm{~V}$. Based on the results presented, it is conclude that low resolutions has higher number of samples which more accurate in term of the sampling signal.

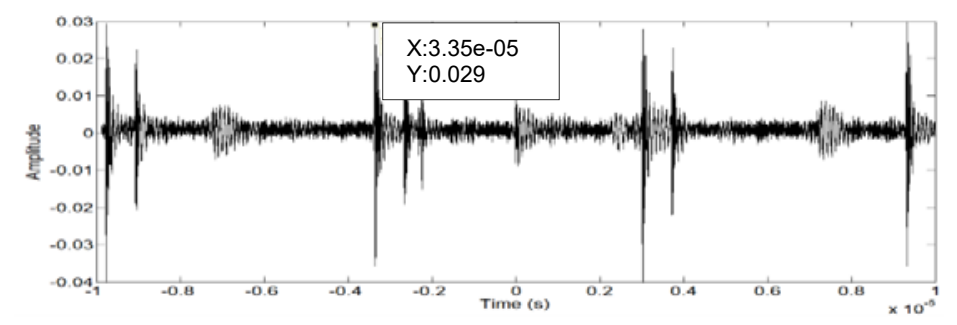

Figure 6. PD signal measured from on-site measurement using RC

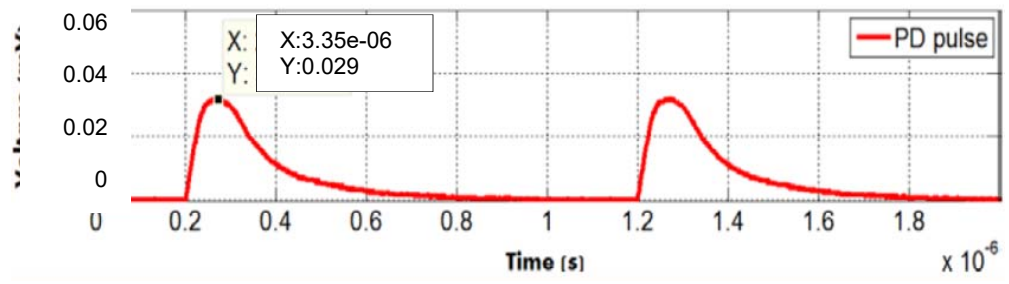

Figure 7. Peak PD pulse from on-site measurement 


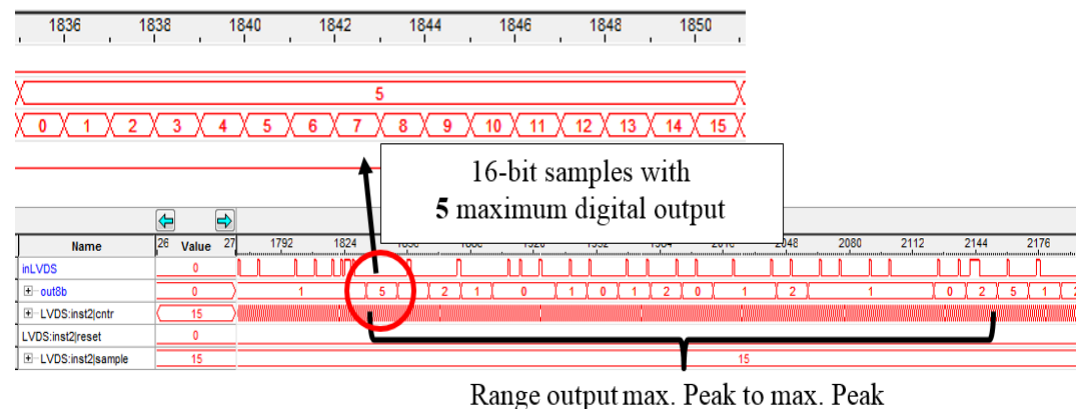

Figure 8. 4-bit resolution sampling signal for PD measurement

Figure 9 represents, the complete GUI for smart online PD monitoring system where it capable to shows the detected online PD signal in real time condition depend on three located RC sensors and the distance of PD signal detected was automated calculate and indicate in this platform. Result indicates that the peak amplitude of PD signal within 4.3 metre detected along $5 \mathrm{~km} 11 \mathrm{kV}$ power cable. Although the result indicate several different in term of peak amplitude, but the figure of peak and value still significant to be used. Three exact location of the PD is displayed individually depend on sensor A, B and C located at power cable. Comparison between three sensor location indicated that the nearest PD signal is exist at 1.3-meter form sensor B. Next, the maintenance unit can easily perform their task to solve this problem. Therefore, the feasibility and validity of this monitoring system were verified through real on-site measurement.

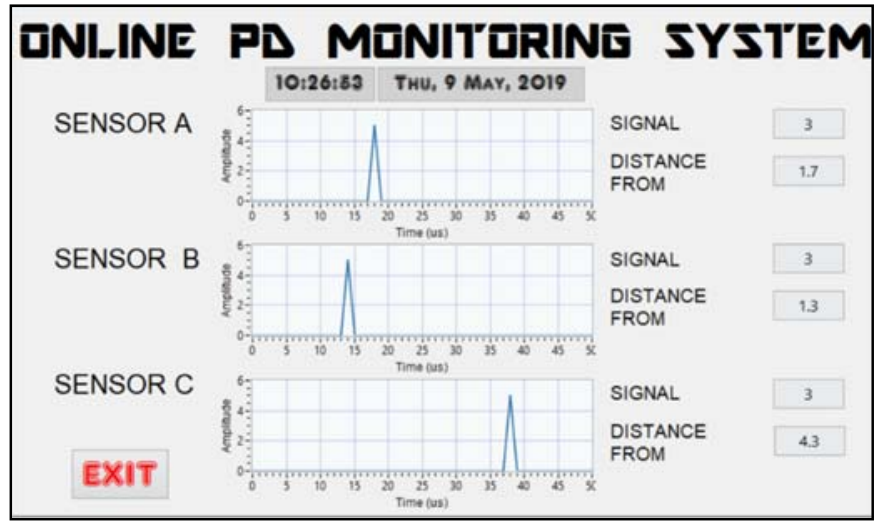

Figure 9. The complete GUI of online PD monitoring system

\section{CONCLUSION}

This paper presents the development of smart online PD monitoring system for medium voltage power cable. The RC sensor's developed function as a powerful component for PD detection was employed with wireless system using raspberry pi and monitored by smart interface using LabVIEW making the system valuable for on-line of a power cable. It shows that the PD signal in power cable can be detected by the RC sensor and notify the user or utility wirelessly by the monitoring system. Finally, the application of smart online PD monitoring can be applied and will help to improve the capability of PD detection in the medium voltage power cable.

\section{ACKNOWLEDGEMENTS}

The authors gratefully acknowledge Universiti Malaysia Perlis (UniMAP) for the equipment support, Research Management Centre, UniMAP through research grant of Internal Sosial Project Via Research (INSPIRE) 9001-00581 for financial support and to the Ministry of Higher Education for their support through the SLAB scheme.

\section{REFERENCES}

Development of smart online partial discharge monitoring system for medium ... (A. Z. Abdullah) 
[1] M. H. Jopri, A. R. Abdullah, T. Sutikno, M. Manap, M. R. A. Ghani, and M. R. Yusoff, "A Critical Review of Time-frequency Distribution Analysis for Detection and Classification of Harmonic Signal in Power Distribution System," International Journal of Electrical and Computer Engineering (IJECE), vol. 8, no. 6, pp. 4603-4618, 2018.

[2] M. Shafiq, L. Kutt, M. Lehtonen, T. Nieminen, and M. Hashmi, "Parameters identification and modeling of highfrequency current transducer for partial discharge measurements," IEEE Sens. J., vol. 13, no.3, pp. 1081-1091, 2013.

[3] L. Renforth, R. Giussani, M. T. Mendiola, and L. Dodd, "On-Line Partial Discharge Insulation Condition Monitoring of Complete High Voltage Networks," IEEE Trans. Ind. Appl., vol. 9994, no. c, pp. 1-9, 2018.

[4] R. N. Wu and C. K. Chang, "The use of partial discharges as an online monitoring system for underground cable joints," IEEE Trans. Power Deliv., vol. 26, no. 3, pp. 1585-1591, 2011.

[5] G. C. Montanari, D. Fabiani, P. Morshuis, and L. Dissado, "Why residual life estimation and maintenance strategies for electrical insulation systems have to rely upon condition monitoring," IEEE Trans. Dielectr. Electr. Insul., vol. 23, no. 3, pp. 1375-1385, 2016.

[6] C. Sumereder and M. Muhr, "Estimation of Residual Lifetime - Theory and Practical Problems," vol. 1, no. November, pp. 1-6, 2005.

[7] Muzamir Isa, "Partial Discharge Location Technique for Covered- Conductor Overhead Distribution Lines," Aalto University, 2013.

[8] P. Mulroy, A. Hurtado, and D. Badetz, "On-line partial discharge monitoring system for distribution networks," Proc. 2012 IEEE Int. Conf. Cond. Monit. Diagnosis, C. 2012, no. September, pp. 542-545, 2012.

[9] D. A. Genutis, "Neta World-Using PD surveys to increase electrical reliability," 2001.

[10] M. Shafiq, G. A. Hussain, L. Kütt, N. I. Elkalashy, and M. Lehtonen, "Partial discharge diagnostic system for smart distribution networks using directionally calibrated induction sensors," Electr. Power Syst. Res., vol. 119, pp. 447-461, 2015.

[11] S. H. K. Hamadi et al., "Modelling of partial discharge signal and noise interference using labview," IEEE Student Conf. Res. Dev. Inspiring Technol. Humanit. SCOReD 2017 - Proc., vol. 2018-Janua, pp. 451-455, 2018.

[12] H. N. Mohd Shah et al., "Develop and implementation of PC based controller for humanoid robot using digital potentiometer," Indonesian Journal of Electrical Engineering and Computer Science, vol. 15, no. 1, p. 104, 2019.

[13] N. A. Salim, H. Mohamad, Z. M. Yasin, N. Fadilah, and A. Aziz, "Graphical user interface based model for transmission line performance implementation in power system," vol. 16, no. 1, pp. 92-100, 2019.

[14] N. Telagam, S. Lakshmi, and K. Nehru, "Ber analysis of concatenated levels of encoding in GFDM system using labview," Indonesian Journal of Electrical Engineering and Computer Science, vol. 14, no. 1, pp. 80-91, 2019.

[15] M. N. K. H. Rohani et al., "Evaluation of Rogowski coil sensor performance using EMTP-ATP software," in 2016 3rd International Conference on Electronic Design, ICED 2016, 2017, pp. 446-451.

[16] R. Schwarz and M. Muhr, "Modern technologies in optical partial discharge detection," Annu. Rep. - Conf. Electr. Insul. Dielectr. Phenomena, CEIDP, pp. 163-166, 2007.

[17] A. Z. Abdullah, M. Isa, S. N. M. Arshad, M. N. K. H. Rohani, and H. S. A. Halim, "Wavelet Based De-Noising for On-Site Partial Discharge Measurement Signal,” Indones. J. Electr. Eng. Comput. Sci., vol. 11, no. 1, pp. 7-15, 2019.

[18] A. Z. Abdullah and M. Isa, "Modelling of Partial Discharge Measurement Using Rogowski Coil As New Approach," in eProceedings National Innovation and Invention Competition Through Exhibition 2018, 2018.

[19] M. N. K. H. Rohani, M. Isa, M. Syahril, C. C. Yii, A. S. Rosmi, and B. Ismail, "Sigma-Delta ADC Topology Implementation Based on Partial Discharge Detection using Rogowski Coil Sensor,” J. Phys. Conf. Ser., vol. 1019, no. $1,2018$.

[20] A. Z. Abdullah, M. N. K. H. Rohani, M. Isa, H. Hamid, S. N. M. Arshad, and M. Othman, "Real On-Site Partial Discharge Measurement Technique in Medium Voltage Power Cable," 2018 IEEE 7th Int. Conf. Power Energy, PECon 2018, no. 1, pp. 405-408, 2019.

[21] M. H. Samimi, A. Mahari, M. A. Farahnakian, and H. Mohseni, "The rogowski coil principles and applications: A review," IEEE Sensors Journal, vol. 15, no. 2. pp. 651-658, 2015.

[22] A. Z. Abdullah, M. Isa, M. N. K. H. Rohani, M. Othman, and H. A. Hamid, "Measurement and Analysis of Partial Discharge for Medium Voltage Underground Power Cable," vol. 2018, no. November, pp. 1-2, 2018.

[23] M. N. K. H. Rohani et al., "Geometrical Shapes Impact on the Performance of ABS-Based Coreless Inductive Sensors for PD Measurement in HV Power Cables," IEEE Sens. J., vol. 16, no. 17, pp. 6625-6632, 2016.

[24] F. Nugroho and A. B. Pantjawati, "Sigma-Delta ADC Topology Implementation Based on Partial Discharge Detection using Rogowski Coil Sensor" 2018.

[25] C. C. Yii, M. N. K. H. Rohani, M. Isa, S. I. S. Hassan, B. Ismail, and N. Hussin, "Multi-end Partial Discharge Location Algorithm based on Trimmed Mean Data Filtering Technique for MV Underground Cable," pp. 345-350, 2015.

Int J Pow Elec \& Dri Syst Vol. 10, No. 4, Dec 2019 : 2190 - 2197 


\section{BIOGRAPHIES OF AUTHORS}
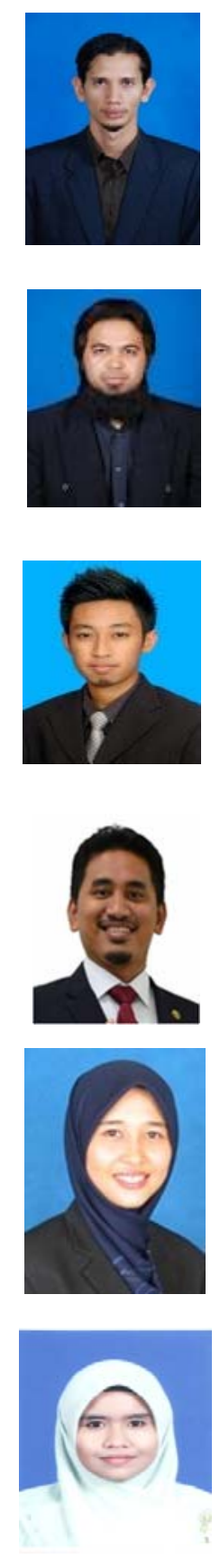

Ahmad Zaidi Bin Abdullah was born in Kedah, Malaysia in 1981. He received his B.Eng (Hons.) degree in Industrial Electronic Engineering from University Malaysia Perlis and M.Eng in Electrical Power from University Tenaga Nasional. He is currently pursuing a $\mathrm{PhD}$ at Universiti Malaysia Perlis. He is a member of Board Engineer Malaysia and Institute Engineer Malaysia. His main research interest are high-voltage engineering, power transformer and partial discharge on-line monitoring.

Muzamir Bin Isa was born in Perlis, Malaysia in 1979. He received his B.Eng (Hons.) degree in Electrical Engineering from University Teknologi Malaysia, M.Eng in Electrical Power from University Tun Hussein Onn Malaysia, and PhD in Power System and High Voltage Engineering from Aalto University, Finland. He is a member of the Board Engineer Malaysia. His main research interest are high-voltage engineering, power transformer and partial discharge on-line monitoring.

Mohamad Nur Khairul Hafizi Rohani was born in Johor, Malaysia, in 1989. He received the B.Eng. (Hons.) degree in industrial electronic engineering and $\mathrm{PhD}$ from the University Malaysia Perlis, Malaysia, in 2013. His research interests is the design of sensors for online condition components based on partial discharge measurement.

Syed Akhmal b Syed Jamalil is a Lecturer in Electrical Department, Faculty of Engineering Technology, University Malaysia Perlis. He teaches Power Electronics and his last research focuses on Solving Dispatch Problem using Particle Swarm Optimization. He received a B.Eng. (Hons.) degree in Industrial Electronic Engineering, Universiti Malaysia Perlis and M.Eng of Electrical Engineering from Universiti Tun Hussein Onn, Johor, Malaysia

Ami Nurul Nazifah Abdullah is a lecturer in School of Electrical System Engineering, University Malaysia Perlis. She teaches Electrical Machine and her last research focuses on Electrical Machine and Renewable Energy. She received a B.Eng. (Hons.) degree in Industrial Electronic Engineering, Universiti Malaysia Perlis and M.Eng of Electrical Engineering from Universiti Putra, Malaysia.

Nurhafiza bt Azizan was born in Pulau Pinang, Malaysia in 1982. She received a B.Eng. (Hons.) degree in Industrial Electronic Engineering and a Master of Science in Electrical Power Engineering from University Malaysia Perlis, Malaysia. She has worked as vocational traning official at faculty of engineering technology. Registered as professional technologies (Ts), her research interests include power systems and energy management systems. 